\title{
Über die Löslichkeit des Berylliumsulfats in Wasser und Schwefelsäure.
}

\author{
Von \\ F. Wirth.
}

Mit 1 Figur im Text.

Über die Hydrate des Berylliumsulfats sind wir durch die Arbeiten von Parsons $^{1}$ sowie von Levr Malvano ${ }^{2}$ unterrichtet. Parsons untersuchte die Fähigkeit der Sulfatlösungen Berylliumhydroxyd aufzulösen, Levi MaLvano stellte die Existenzbedingungen sowie die Umwandlungspunkte der verschiedenen normalen Hydrate fest. Nach ihm liegt der Umwandlungspunkt des 6-Hydrats in das Bihydrat bei $77.4^{\circ}$, der des 4-Hydrats in das Bihydrat bei $111.5^{\circ}$. Dies geht dann bei $158^{\circ}$ in das Monohydrat über.

Levi Malvano hat die Löslichkeit der verschiedenen Hydrate in Wasser bestimmt. Wie schon Ephraim in der neven Bearbeitung des GMeLin KRAUT ${ }^{3}$ hervorhob, sind diese Bestimmungen nicht einwandfrei, da der Autor das $\mathrm{BeSO}_{4} .4 \mathrm{H}_{2} \mathrm{O}$ aus Lösungen gewinnt, welche überschüssige Schwefelsäure enthalten. Die ron ihm angeführten Analysen stimmen auch nicht auf $\mathrm{BeSO}_{4} .4 \mathrm{H}_{2} \mathrm{O}$, für welchen Körper er fälschlich $14.17 \% \mathrm{BeO}, 54.20 \% \mathrm{SO}_{3}$ und $31.63 \% \mathrm{H}_{2} \mathrm{O}$ berechnet. Levi Malvano findet, daB bei $31^{\circ}$ auf $1 \mathrm{Mol} \mathrm{BeSO}_{4}$ 11.18 Mole Wasser kommen. Nach den übrigen Werten kann für $25^{\circ}$ auf $1 \mathrm{Mol} \mathrm{BeSO}_{4} 11.5$ Mole Wasser berechnet werden. Demnach können auf $100 \mathrm{~g}$ Wasser $50.78 \mathrm{~g} \mathrm{BeSO}_{4}$ und in $100 \mathrm{~g}$ Flüssigkeit sollten $33.67 \mathrm{~g} \mathrm{BeSO}_{4}=0.3202 \mathrm{Mol}$ Sulfatanhydrid gelöst sein.

Diese Werte entsprechen nach meinen Untersuchungen nicht den Tatsachen. Sie sind viel zu hoch. Ich verwandte zu meinen Bestimmungen reinstes Berylliumsulfat-6-Hydrat, das ich nach Berzelius mehrmals durch Fällen mit Alkohol reinigte. Die Analyse ergab folgende Zusammensetzung:

$1 Z$. anorg. Chem. 40, 409 und 42, 250.

2. anorg. Chem. 48, 451.

3 II 2, S. 533 .

Z. anorg. Chem. Bd, 79 . 
Berechnet für $\mathrm{BeSO}_{4} \cdot 6 \mathrm{H}_{2} \mathrm{O}$ :

Gefunden:

$\begin{array}{lllc}\mathrm{BeO} & 11.77 \% & 12.19 \% & 11.99 \% \\ \mathrm{SO}_{3} & 37.53 \% & 37.49 \% & - \\ \mathrm{H}_{2} \mathrm{O} & 50.70 \% & 50.58 \% & -\end{array}$

Zuerst wurde die Löslichkeit des Hexahydrats in Wasser bestimmt. Ich fand, daß bei $25^{\circ}$ in $100 \mathrm{~g}$ Flüssigkeit $1.96 \mathrm{~g} \mathrm{BeO}=$ $8.212 \mathrm{~g} \mathrm{BeSO}_{4}$ gelöst sind. Eine zweite Löslichkeitsbestimmung, bei der die Temperatur ron oben her erreicht wurde, ergab $1.984 \mathrm{~g} \mathrm{BeO}$.

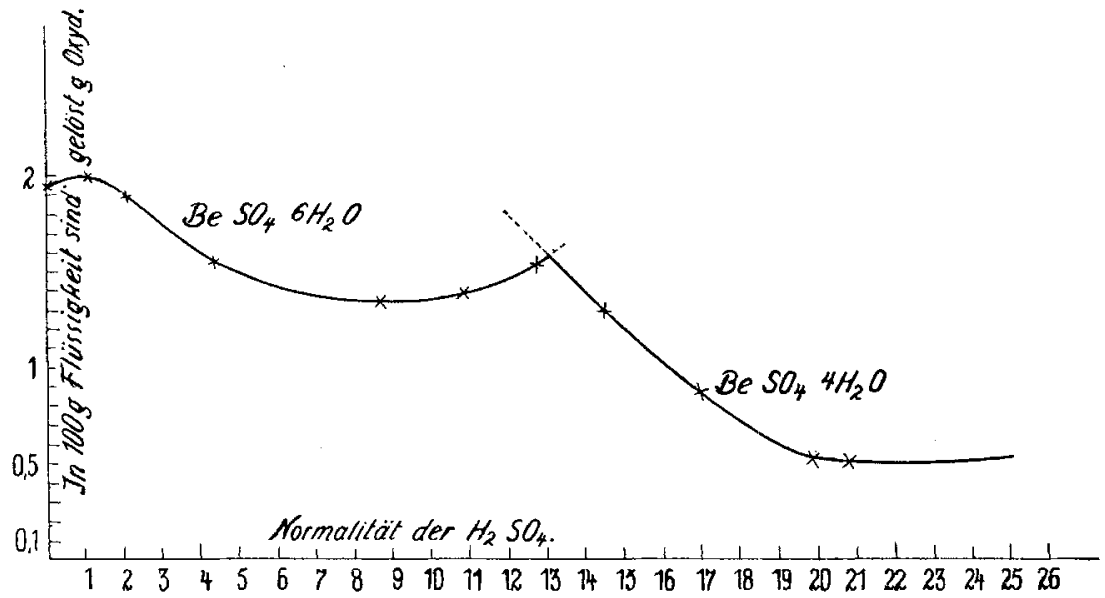

Daraus ergibt sich als Mittelwert $1.972 \mathrm{~g} \mathrm{BeO}$. Es sind also in $100 \mathrm{~g}$ Flüssigkeit $8.262 \mathrm{~g} \mathrm{BeSO}_{4}$, d. i. $0.07857 \mathrm{~g}$-Mol Sulfatanhydrid gelöst, nicht $0.3202 \mathrm{Mol}$, wie sich aus den Werten, die Lnvi Malvano fand, berechnen läßt.

Über das Verhalten des Hexahydrats zu Schwefelsäure liegen noch keine bestimmten Angaben vor. Wie aus Tabelle und Diagramm hervorgeht, wird die Löslichkeit des Sulfats durch Schwefelsäure nur wenig erniedrigt. Wendet man stärkere Säuren an $(n>12.5)$, so werden die fast durchsichtigen großen Hexahydratkristalle sofort trübe und zerfallen unter Bildung von Tetrahydrat in ein lockeres weiBes Kristallpulver, das sich nur sehr schwer absetzt. Die Löslichkeit des Berylliumsulfattetrahydrats $\mathrm{BeSO}_{4} .4 \mathrm{H}_{2} \mathrm{O}$ wird durch Schwefelsäure stärker beeinfluBt. Die Löslichkeit in einer 20-norm. Säure beträgt etwa den vierten Teil der Wasserlöslichkeit. Nachstehende Tabelle gibt die genauen Resultate. 
Löslichkeit des Berylliumsulfats in Wasser und Schwefelsäure. 359

Löslichkeit des Berylliumsulfats in Schwefelsäure bei $25^{\circ}$.

\begin{tabular}{|c|c|c|c|c|c|c|}
\hline \multirow{2}{*}{$\begin{array}{l}\text { Ver- } \\
\text { suchs- } \\
\mathrm{Nr} \text {. }\end{array}$} & \multicolumn{2}{|c|}{$\begin{array}{l}\text { Angewandte } \\
\text { Säure }\end{array}$} & \multicolumn{2}{|c|}{$\begin{array}{c}\text { In } 100 \mathrm{~g} \text { Flüssigkeit } \\
\text { sind gelöst }\end{array}$} & \multirow{2}{*}{$\begin{array}{l}\text { In } 1000 \text { g Flüs- } \\
\text { sigkeit sind ge- } \\
\text { löst } \mathrm{g}-\mathrm{Mol} \\
\text { Sulfatanhydrid }\end{array}$} & \multirow{2}{*}{$\begin{array}{l}\text { Boden- } \\
\text { körper }\end{array}$} \\
\hline & $\begin{array}{c}\text { Norma- } \\
\text { lität }\end{array}$ & $\begin{array}{c}\mathrm{H}_{2} \mathrm{SO}_{4} \\
\%\end{array}$ & $\begin{array}{c}\mathrm{BeO} \\
\mathrm{g}\end{array}$ & $\begin{array}{c}\mathrm{BeSO}_{4} \\
\mathbf{g}\end{array}$ & & \\
\hline 1 & Wasser & - & 1.96 & 8.212 & 0.7809 & $\mathrm{BeSO}_{4} .6 \mathrm{H}_{2} \mathrm{O}$ \\
\hline 2 & 1.1 & 5.23 & 2.012 & 8.429 & 0.8016 & $"$ \\
\hline 3 & 2.11 & 9.61 & 1.896 & 7.944 & 0.7554 & $"$ \\
\hline 4 & 4.32 & 18.70 & 1.576 & 6.603 & 0.6279 & $n$ \\
\hline 5 & 8.7 & 34.00 & 1.344 & 5.631 & 0.5355 & $"$ \\
\hline 6 & 10.8 & 40.35 & 1.378 & 5.773 & 0.5490 & , \\
\hline 7 & 12.6 & 45.51 & 1.582 & 6.628 & 0.6303 & , \\
\hline 8 & 14.5 & 50.63 & 1.298 & 5.438 & 0.5171 & $\mathrm{BeSO}_{4} .4 \mathrm{H}_{2} \mathrm{O}$ \\
\hline 9 & 16.96 & 56.59 & 0.8689 & 3.640 & 0.3462 & $"$ \\
\hline 10 & 19.84 & 63.24 & 0.5356 & 2.244 & 0.2134 & $"$ \\
\hline 11 & 20.78 & 65.24 & $0.507_{8}$ & 2.128 & 0.2023 & $"$ \\
\hline 12 & 24.92 & 73.64 & 0.5214 & 2.185 & 0.2077 & $"$ \\
\hline
\end{tabular}

Da die Löslichkeitskurve des Aluminiumsulfats in Schwefelsäure ganz anders verläuft, kann das Verhalten des Berylliumsulfats zu Schwefelsäure zur letzten Reinigung dieses Elementes benutzt werden, zumal reine Berylliumverbindungen, sowie reines Berylliummetall ${ }^{1}$ auch in der Technik gebraucht werden.

1 Berylliumnitrat wird z. B. als Härtemittel in der Glühstrumpfindustrie verwandt. Berylliummetall und besonders Legierungen dieses Elementes z. B. mit Wolfram haben gute metallurgische Eigenschaften, lassen sich schmieden und zu Blech aushämmern (Verwendung - auch als Hilfsmetall - zu Fäden und Drähten in elektrischen Lampen).

Charlottenburg, Anorg.-chem. Institut. der kgl. techn. Hochschule zu Berlin.

Bei der Redaktion eingegangen am 17. Dezember 1912. 